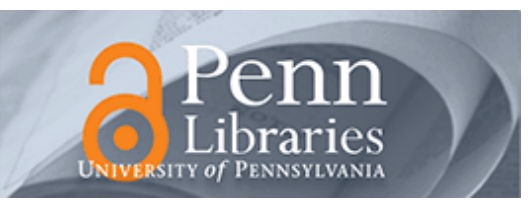

University of Pennsylvania

ScholarlyCommons

April 2007

\title{
Distributed Geodesic Control Laws for Flocking of Nonholonomic Agents
}

\author{
Nima Moshtagh \\ University of Pennsylvania \\ Ali Jadbabaie \\ University of Pennsylvania, jadbabai@seas.upenn.edu
}

Follow this and additional works at: https://repository.upenn.edu/ese_papers

\section{Recommended Citation}

Nima Moshtagh and Ali Jadbabaie, " Distributed Geodesic Control Laws for Flocking of Nonholonomic Agents", . April 2007.

Copyright 2007 IEEE. Reprinted from IEEE Transactions on Automatic Control, Volume 52, Issue 4, April 2007, pages 681-686. Publisher URL: http://dx.doi.org/10.1109/TAC.2007.894528

This material is posted here with permission of the IEEE. Such permission of the IEEE does not in any way imply IEEE endorsement of any of the University of Pennsylvania's products or services. Internal or personal use of this material is permitted. However, permission to reprint/republish this material for advertising or promotional purposes or for creating new collective works for resale or redistribution must be obtained from the IEEE by writing to pubs-permissions@ieee.org. By choosing to view this document, you agree to all provisions of the copyright laws protecting it.

This paper is posted at ScholarlyCommons. https://repository.upenn.edu/ese_papers/239

For more information, please contact repository@pobox.upenn.edu. 


\title{
Distributed Geodesic Control Laws for Flocking of Nonholonomic Agents
}

\begin{abstract}
We study the problem of flocking and velocity alignment in a group of kinematic nonholonomic agents in 2 and 3 dimensions. By analyzing the velocity vectors of agents on a circle (for planar motion) or sphere (for 3-D motion), we develop a geodesic control law that minimizes a misalignment potential and results in velocity alignment and flocking. The proposed control laws are distributed and will provably result in flocking when the underlying proximity graph which represents the neighborhood relation among agents is connected. We further show that flocking is possible even when the topology of the proximity graph changes over time, so long as a weaker notion of joint connectivity is preserved.
\end{abstract}

\section{Keywords}

cooperative control, distributed coordination, flocking, multiagent systems

\section{Comments}

Copyright 2007 IEEE. Reprinted from IEEE Transactions on Automatic Control, Volume 52, Issue 4, April 2007, pages 681-686. Publisher URL: http://dx.doi.org/10.1109/TAC.2007.894528

This material is posted here with permission of the IEEE. Such permission of the IEEE does not in any way imply IEEE endorsement of any of the University of Pennsylvania's products or services. Internal or personal use of this material is permitted. However, permission to reprint/republish this material for advertising or promotional purposes or for creating new collective works for resale or redistribution must be obtained from the IEEE by writing to pubs-permissions@ieee.org. By choosing to view this document, you agree to all provisions of the copyright laws protecting it. 
[13] C. C. White, "Bonds on optimal cost for a replacement problem with partial observation," Naval Res. Logist. Quart., vol. 26, pp. 415-422, 1979.

[14] S. Yoshikazu and Y. Tsuneo, "Discrete-time Markovian decision processes with incomplete state observation," Ann. Math. Statist., vol. 41, pp. 78-86, 1970.

[15] Q. C. Zhao, Y. C. Ho, and Q. S. Jia, "Vector ordinal optimization," $J$. Optim. Theory Appl., vol. 125, pp. 259-274, 2005.

\section{Distributed Geodesic Control Laws for Flocking of Nonholonomic Agents}

\author{
Nima Moshtagh and Ali Jadbabaie
}

\begin{abstract}
We study the problem of flocking and velocity alignment in a group of kinematic nonholonomic agents in 2 and 3 dimensions. By analyzing the velocity vectors of agents on a circle (for planar motion) or sphere (for 3-D motion), we develop a geodesic control law that minimizes a misalignment potential and results in velocity alignment and flocking. The proposed control laws are distributed and will provably result in flocking when the underlying proximity graph which represents the neighborhood relation among agents is connected. We further show that flocking is possible even when the topology of the proximity graph changes over time, so long as a weaker notion of joint connectivity is preserved.
\end{abstract}

Index Terms-Cooperative control, distributed coordination, flocking, multiagent systems.

\section{INTRODUCTION}

Cooperative control of multiple autonomous agents has become a very active part of control theory research. The main underlying theme of this line of research is to analyze and/or synthesize spatially distributed control architectures that can be used for motion coordination of large groups of autonomous vehicles. Each vehicle is assumed to be capable of local sensing and communication, and there is often a global objective, such as swarming, or reaching a stable formation, etc. A nonexhaustive list of relevant research in control theory and robotics includes [1], [3], [5], [8]-[10], [12], [13], [19].

On the other hand, such problems of distributed coordination have also been studied in areas as diverse as statistical physics and dynamical systems (in the context of synchronization of oscillators and alignment of self propelled particles [18]), in biology, and ecology, and in computer graphics in the context of artificial life and simulation of social aggregation phenomena, and in distributed computation [17], in the context of reaching consensus in parallel and distributed processing.

Most of the above cited research on distributed control of multivehicle systems has been focused on fully actuated systems [16], or planar

Manuscript received September 16, 2005; revised March 10, 2006. Recommended by Associate Editor F. Bullo. This work was supported in part by the following grants: ARO-MURI Grant W911NF-05-1-0381, ONR YIP N00014-04-1-046 and ONR N00014-06-1-0436, and NSF-ECS-0347285.

The authors are with the department of Electrical and Systems Engineering and GRASP Laboratory, University of Pennsylvania, Philadelphia, PA 19104 USA (e-mail: nima@grasp.upenn.edu; jadbabai@grasp.upenn.edu).

Color versions of one or more of the figures in this paper are available online at http://ieeexplore.ieee.org.

Digital Object Identifier 10.1109/TAC.2007.894528 under-actuated systems [8]. Our goal here is to develop motion coordination algorithms that can be used for distributed control of a group of nonholonomic vehicles in 2 and 3 dimensions. Using results of Bullo et al. [2] we develop geodesic control laws that result in flocking and velocity alignment for nonholonomic agents in 3 dimensions.

In order to introduce the idea of a geodesic control law to the reader, we start with the special case of planar motion in Section III. We will show that the planar version of such a control law (where the velocity vector is restricted to stay on a circle) is exactly the well-known Kuramoto model of coupled nonlinear oscillators [6], [14]. Such a control law is a gradient controller that minimizes a potential function which represents the aggregate "misalignment energy" between all agents. In Section V we return to the general case of 3-D motion and we develop control laws that result in stable coordination and velocity alignment of a group of agents with a fixed connectivity graph. In Section VI, we show that flocking is possible even when the topology of the proximity graph changes over time. Finally, in Section VII, we provide simulations that show the effectiveness of the designed controllers. But, let us review the concepts of graph theory that we use in this note for stability analysis.

\section{GRAPH THEORY PRELIMINARIES}

In this section, we introduce some standard graph theoretic notation and terminology. An (undirected) graph $\mathbb{G}$ consists of a vertex set, $\mathcal{V}$, and an edge set $\mathcal{E}$, where an edge is an unordered pair of distinct vertices in $\mathbb{G}$. If $x, y \in \mathcal{V}$, and $(x, y) \in \mathcal{E}$, then $x$ and $y$ are said to be adjacent, or neighbors and we denote this by writing $x \sim y$. The number of neighbors of each vertex is its valence. A path of length $r$ from vertex $x$ to vertex $y$ is a sequence of $r+1$ distinct vertices starting with $x$ and ending with $y$ such that consecutive vertices are adjacent. If there is a path between any two vertices of a graph $\mathbb{G}$, then $\mathbb{G}$ is said to be connected. If there is such a path on a directed graph ignoring the direction of the edges, then the graph is weakly connected.

The adjacency matrix $A(\mathbb{G})=\left[a_{i j}\right]$ of an (undirected) graph $\mathbb{G}$ is a symmetric matrix with rows and columns indexed by the vertices of $\mathbb{G}$, such that $a_{i j}=1$ if vertex $i$ and vertex $j$ are neighbors and $a_{i j}=0$, otherwise. The valence matrix $D(\mathbb{G})$ of a graph $\mathbb{G}$ is a diagonal matrix with rows and columns indexed by $\mathcal{V}$, in which the $(i, i)$-entry is the valence of vertex $i$. The (un)directed graph of a (symmetric) matrix is a graph whose adjacency matrix is constructed by replacing all nonzero entries of the matrix with 1 . Matrix $A$ has property $S C$ if and only if $|A|$ is the adjacency matrix of a strictly connected graph.

The symmetric singular matrix defined as:

$$
L(\mathbb{G})=D(\mathbb{G})-A(\mathbb{G})
$$

is called the Laplacian of $\mathbb{G}$. The Laplacian matrix captures many topological properties of the graph. The Laplacian $L$ is a positive semidefinite $M$-matrix (a matrix whose off-diagonal entries are all nonpositive) and the algebraic multiplicity of its zero eigenvalue (i.e., the dimension of its kernel) is equal to the number of connected components in the graph. The $n$-dimensional eigenvector associated with the zero eigenvalue is the vector of ones, $\mathbf{1}$.

Given an orientation of the edges of a graph, we can define the incidence matrix of the graph to be a matrix $B$ with rows indexed by vertices and columns indexed by edges with entries of 1 representing the source of a directed edge and -1 representing the sink. The Laplacian matrix of a graph can also be represented in terms of its incidence matrix as $L=B B^{T}$ independent of the orientation of the edges. 


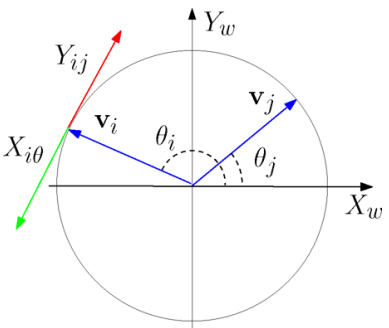

Fig. 1. Velocity vectors of agent $i$ and its neighbor $j$ are projected on the unit circle. $X_{i \theta}$ is the tangent vector to $\mathbf{v}_{i}$.

\section{Distributed CONTROL of Planar Nonholonomic Vehicles}

Consider a group of $N$ agents on a plane. Each agent is capable of sensing some information from its neighbors as defined by

$$
\mathcal{N}_{i} \doteq\{j \mid i \sim j\} \subseteq\{1, \ldots, N\} \backslash\{i\} .
$$

The neighborhood set of agent $i, \mathcal{N}_{i}$, is a set of agents that can share their heading (orientation) information with agent $i$. The size of the neighborhood depends on the characteristics of the communication device. We therefore assume that there is a predetermined radius $R$ which determines the neighborhood relationship. The location of agent $i,(i=$ $1, \ldots, N)$ in the world coordinates is given by $\left(x_{i}, y_{i}\right)$ and its velocity is $\mathbf{v}_{i}=\left(\dot{x}_{i}, \dot{y}_{i}\right)^{T}$. The heading or orientation of agent $i$ is $\theta_{i}$ and is given by: $\theta_{i}=\operatorname{atan} 2\left(\dot{y}_{i}, \dot{x}_{i}\right)$.

It is assumed that all agents move with constant unit speed. Thus, the kinematic model of each agent can be written as

$$
\begin{aligned}
& \dot{x}_{i}=\cos \theta_{i} \\
& \dot{y}_{i}=\sin \theta_{i} \\
& \dot{\theta}_{i}=\omega_{i} \quad i=1, \ldots, N .
\end{aligned}
$$

The goal is to design the control input $\omega_{i}$ so that the headings of the mobile agents reach agreement and velocity vectors are aligned. We therefore define the consensus set as follows.

Definition 3.1: The set of states where the headings of all agents are the same is called the consensus set.

In this note, we say agents are "flocking" when their headings reach asymptotic agreement. We consider the case where the neighboring relations among agents are represented by a fixed weighted graph.

Definition 3.2: The proximity graph $\mathbb{G}=\{\mathcal{V}, \mathcal{E}, \mathcal{W}\}$ is a weighted graph consisting of

- a set of vertices $\mathcal{V}$ indexed by the set of mobile agents;

- a set of edges $\mathcal{E}=\left\{(i, j) \mid \mathbf{v}_{i}, \mathbf{v}_{j} \in \mathcal{V}\right.$, and $\left.i \sim j\right\}$;

- a set of weights $\mathcal{W}$, over the set of edges $\mathcal{E}$.

In order to design the desired control law for agent $i$, let us view all the velocity vectors of neighbors of agent $i$ in a unit circle as shown in Fig. 1. Each velocity vector $\mathbf{v}_{i}$ can be written in terms of the heading angle $\theta_{i}$ (measured in a fixed inertial frame) as follows:

$$
\mathbf{v}_{i}=\left(\begin{array}{c}
\cos \theta_{i} \\
\sin \theta_{i}
\end{array}\right), i=1, \ldots, N
$$

As the velocity vector $\mathbf{v}_{i}$ changes, we can write the dynamics equation corresponding to the motion of agent $i$ as $\dot{\mathbf{v}}_{i}=\omega_{i} X_{i \theta}$ where vector $X_{i \theta}$ is tangent to $\mathbf{v}_{i}$ and is given by

$$
X_{i \theta}=\left(\begin{array}{c}
-\sin \theta_{i} \\
\cos \theta_{i}
\end{array}\right), \quad i=1, \ldots, N
$$

Note: In the following the standard inner product is denoted by $\langle\cdot, \cdot\rangle$, and the cross product by $\times$.

Let $\alpha_{i j}$ be the angle between two velocity vectors $\mathbf{v}_{i}$ and $\mathbf{v}_{j}, \alpha_{i j}=$ $\left|\theta_{i}-\theta_{j}\right|$. When $\mathbf{v}_{i}$ and $\mathbf{v}_{j}$ are neither equal nor opposite $\left(0<\alpha_{i j}<\right.$ $\pi$ ), we can define a unit vector $Y_{i j}$ tangent to $\mathbf{v}_{i}$ such that it is pointing towards the velocity vector $\mathbf{v}_{j}$. This unit-length vector is defined as

$$
Y_{i j} \doteq \frac{\mathbf{v}_{j}^{\perp}}{\left|\mathbf{v}_{j}^{\perp}\right|}=\frac{\left(\mathbf{v}_{i} \times \mathbf{v}_{j}\right) \times \mathbf{v}_{i}}{\left\|\left(\mathbf{v}_{i} \times \mathbf{v}_{j}\right) \times \mathbf{v}_{i}\right\|}=\frac{\mathbf{v}_{j}-\left\langle\mathbf{v}_{i}, \mathbf{v}_{j}\right\rangle \mathbf{v}_{i}}{\sin \alpha_{i j}}
$$

where $\mathbf{v}_{j}^{\perp}$ is the component of $\mathbf{v}_{j}$ orthogonal to $\mathbf{v}_{i}$. Now, we can prove the following theorem for the distributed control of the velocity vectors of a group of $N$ agents.

Theorem 3.3: Consider the system of $N$ equations $\dot{\mathbf{v}}_{i}=\omega_{i} X_{i \theta}, i=$ $1, \ldots, N$. If the proximity graph is fixed and connected, then by applying the control law

$$
\omega_{i}=\sum_{j \in \mathcal{N}_{i}} \sin \alpha_{i j}\left\langle Y_{i j}, X_{i \theta}\right\rangle=\sum_{j \in \mathcal{N}_{i}}\left\langle\mathbf{v}_{j}, X_{i \theta}\right\rangle
$$

all trajectories converge to the set of equilibrium points given by $\dot{\theta}=0$. Furthermore, consensus set is locally attractive.

Proof: We observe that on the unit circle $Y_{i j}=X_{i \theta}$ or $Y_{i j}=$ $-X_{i \theta}$, depending on the orientations of $\mathbf{v}_{i}$ and $\mathbf{v}_{j}$. Hence, we write the input (5) as

$$
\omega_{i}=-\sum_{j \in \mathcal{N}_{i}} \sin \left(\theta_{i}-\theta_{j}\right)
$$

Notice that the above input is exactly the one used in the Kuramoto model of coupled nonlinear oscillators [6], [10], [14].

Assume an arbitrary orientation for the edges of graph $\mathbb{G}$. Consider the $N \times d$ incidence matrix, $B$, of this oriented graph with $N$ vertices and $d$ edges. Then, we can write (6) as

$$
\dot{\theta}=\omega=-B \sin \left(B^{T} \theta\right)
$$

where $\theta=\left[\theta_{1}, \ldots, \theta_{N}\right]^{T}$, and $\sin \left(B^{T} \theta\right) \in \mathbb{R}^{d}$ is a vector whose elements are $\sin \left(\theta_{i}-\theta_{j}\right)$. Equation (7) can be written in a more compact form

$$
\dot{\theta}=\omega=-B W(\theta) B^{T} \theta=-L_{w}(\theta) \theta
$$

where $W(\theta)=\operatorname{diag}\left\{\operatorname{sinc}\left(\theta_{i}-\theta_{j}\right) \mid(i, j) \in \mathcal{E}\right\}$ is a diagonal matrix whose entries are the edge weights for $\mathbb{G}$. The ordering of the elements on the diagonal of $W(\theta)$ is consistent with the ordering of the edges in the incidence matrix $B$. The matrix $L_{w}(\theta)=B W(\theta) B^{T}$ can be thought of as the weighted Laplacian of $\mathbb{G}$, when $\operatorname{sinc}\left(\theta_{i}-\theta_{j}\right)=$ $\sin \left(\theta_{i}-\theta_{j}\right) /\left(\theta_{i}-\theta_{j}\right)$ is positive. For this to hold $\theta$ should belong to the open cube $(-\pi / 2, \pi / 2)^{N}$, where $N$ is the number of vertices of the graph. In other words, over any compact subset of the cube $(-\pi / 2, \pi / 2)^{N}$, the dynamics can be represented by a state-dependent weighted Laplacian.

Now, consider the Lyapunov function

$$
U=\frac{1}{2} \sum_{j \sim i}\left\|\mathbf{v}_{i}-\mathbf{v}_{j}\right\|^{2}=\frac{1}{2}\left[e^{j \theta}\right]^{*} L\left[e^{j \theta}\right]=\sum_{j \sim i} 1-\cos \left(\theta_{i}-\theta_{j}\right)
$$

where the sum is over all the neighboring pairs, denoted by $i \sim j ; L$ is the Laplacian of the graph; and $\left[e^{j \theta}\right]$ is the stack of velocity vectors in 
complex notation. The above sum represents the total misalignment energy between velocity vectors. Since we have $U=d-\mathbf{1}^{T} \cos \left(B^{T} \theta\right)$, and because of (8), the time derivative of $U$ along the trajectories of the system becomes

$$
\dot{U}=\nabla U \dot{\theta}=\theta^{T} L_{w} \dot{\theta}=-\dot{\theta}^{T} \dot{\theta} \leq 0
$$

A simple application of LaSalle's invariance principle over the configuration space which is an $\mathrm{N}$-torus and therefore compact reveals that all trajectories starting in anywhere on the $\mathrm{N}$-torus converge to the largest invariant sets in $E=\{\theta \mid \dot{U}=0\}$. Note that this is a very rich set and contains many equilibria other than the consensus set, some of which could be stable. See Remark 3.4 for an instance of this situation.

In order to prove local attractivity of the consensus set we utilize a simple quadratic Lyapunov function $V=1 / 2\left(\theta^{T} \theta\right)$, and a compact set $\Omega_{c}=\{\theta \mid V \leq c\}$. This set, which is characterized by the largest level set of $V$ that is contained inside the cube $(-\pi / 2, \pi / 2)^{N}$, can be used to show that the synchronized state is the only equilibrium within the set $E=\left\{\theta \in \Omega_{c} \mid \dot{V}=0\right\}$. This is true since $\dot{V}=-\theta^{T} L_{w} \theta \leq 0$. Thus, equilibrium points are the set of solutions of $L_{w} \theta=0$. If graph $\mathbb{G}$ is connected, within $\Omega_{c}$ the null space of weighted Laplacian $L_{w}$ is the span of vector $\mathbf{1} \doteq[1, \ldots, 1]^{T}$. Thus, the solution is $\operatorname{Null}\left\{L_{w}\right\}$, which is the set $S=\{\theta \mid \theta \in \operatorname{span}\{\mathbf{1}\}\}$. This suggests that all agents reach the same heading as $t \rightarrow \infty$.

Remark 3.4: When the proximity graph $\mathbb{G}$ has the ring topology (i.e., all agents have exactly two neighbors), there are two sets of equilibria: $\theta \in \operatorname{span}\{\mathbf{1}\}$ and $B^{T} \theta \in \operatorname{span}\{\mathbf{1}\}$ where the former corresponds to the set $\left\{\theta_{i}=\theta_{j}, \forall i \neq j\right\}$ and the latter corresponds to $\left\{\theta_{i}-\theta_{j}=2 \pi / N, \forall i \neq j\right\}$. See [7] for details.

Remark 3.5: Local attractivity of the consensus set can be established even when the proximity graph changes with time. As will be shown in Section VI, this holds as long as a weak notion of connectivity called joint connectivity [5] holds.

Remark 3.6: The geodesic control input (6) for a group of planar nonholonomic vehicles is basically the same controller that can stabilize the Kuramoto model of coupled nonlinear oscillators [6]. The term $\sin \left(\theta_{i}-\theta_{j}\right)$ in the angular velocity can be explained by noting that in the planar case the angular velocity is the rate of rotation about the axis $\mathbf{v}_{i} \times \mathbf{v}_{j}$ where $\mathbf{v}_{i}$ is given by (3). The norm of $\mathbf{v}_{i} \times \mathbf{v}_{j}$ is nothing but $\sin \left(\theta_{i}-\theta_{j}\right)$.

Remark 3.7: The geodesic controller (6) is the nonlinear version of the control law

$$
\omega_{i}=-\sum_{j \in \mathcal{N}_{i}}\left(\theta_{i}-\theta_{j}\right)
$$

proposed in [5], [12] as the continuous analogue of Viscek's model [18].

\section{LEADER FOLLOWING}

One could envision in a social aggregation scenario such as flocking of birds, one of the flock-mates acts as the leader of the group and others follow the leader while staying in a formation. Similarly, here we consider the case that one additional agent, labeled 0 , acts as the group's leader. Agent 0 moves with the constant unit velocity (same as others) and a fixed heading $\theta_{0}$. Other agents in the group may or may not have the leader as a neighbor. Here, we prove that the control law (6) results in a stable formation of the group while following the leader, so that in the end all agents reach the desired heading $\theta_{0}$ (cf. [5] for more details on leader following).

Consider the input of each agent in the leaderless case that is given by (6). We can separate the leader from other agents and write:

$$
\dot{\theta}_{i}=-\sum_{j \in \mathcal{N}_{i}} \sin \left(\theta_{i}-\theta_{j}\right)-c_{i} \sin \left(\theta_{i}-\theta_{0}\right)
$$

where $c_{i}=1$ if agent $i$ and the leader are neighbors and $c_{i}=0$ otherwise.

To show that all the headings become equal to $\theta_{0}$, we consider the error term $e_{i}=\theta_{i}-\theta_{0}$. Since $\dot{e}_{i}=\dot{\theta}_{i}$, we can write (10) as

$$
\dot{e}_{i}=-\sum_{j \in \mathcal{N}_{i}} \sin \left(e_{i}-e_{j}\right)-c_{i} \sin e_{i}
$$

Consider the error vector $e=\left[e_{1}, \ldots, e_{N}\right]^{T}$. Similar to calculations of Section III, the error dynamics becomes

$$
\dot{e}=-B W(e) B^{T} e-W_{l} e=-L_{w} e-W_{l} e=-H_{l} e
$$

where $W(e)=\operatorname{diag}\left\{\operatorname{sinc}\left(e_{i}-e_{j}\right) \mid(i, j) \in \mathcal{E}\right\} \in \mathbb{R}^{d \times d}$ and $W_{l}=\operatorname{diag}\left\{c_{i} \operatorname{sinc}\left(e_{i}\right) \mid(i, 0) \in \mathcal{E}\right\} \in \mathbb{R}^{N \times N}$. Both $W(e)$ and $W_{l}$ are weight matrices with positive entries, because $\operatorname{sinc}\left(e_{i}-e_{0}\right)=$ $\operatorname{sinc}\left(\theta_{i}-\theta_{0}\right)$ is positive for $\theta_{i} \in(-\pi / 2, \pi / 2)$, and $c_{i}$ is a nonnegative coefficient.

In order to show that the error is asymptotically stable, consider the Lyapunov function $U=1 / 2\left(e^{T} e\right)$. The derivative of this along the trajectory of the error system can be written as $\dot{U}=-e^{T} H_{l} e$, where $H_{l}=L_{w}+W_{l}$. We will prove that $H_{l}$ is positive definite, and the error will asymptotically decay to zero.

Note that both $L_{w}$ and $W_{l}$ are positive-semidefinite matrices and so is $H_{l}$. Matrix $L_{w}$ has property $\mathrm{SC}$, because if we replace the nonzero elements of $L_{w}$ with 1 , we obtain the adjacency matrix of the neighboring graph that is strictly connected. matrix $W_{l}$ is diagonal, thus adding it to $L_{w}$ doesn't change the neighboring graph. Thus $H_{l}=L_{w}+W_{l}$ has property SC. A matrix is irreducible if and only if it has property SC. Thus, $H_{l}$ is irreducible. See [4, Ch. 6], for more details on irreducible matrices.

We need to show that $H_{l}$ is actually positive definite. To do so, we make the following observations.

- $H_{l}$ is an irreducible matrix.

- $L_{w}$ is diagonally dominant.

- For at least one of the rows of $H_{l}$ the diagonal entry is strictly greater than the sum of off-diagonal entries (because $W_{l}$ ia a diagonal matrix with nonnegative entries).

According to Taussky theorem [4] matrix $H_{l}$ is an irreducibly diagonally dominant matrix and is invertible. Thus, $H_{l}$ must be a positive definite matrix. As a result $\dot{U}<0$ and the error vector asymptotically decays to zero; consequently $\theta_{i}=\theta_{0}$ for every $i=1, \ldots, N$, as $t \rightarrow \infty$.

\section{Distributed CoORdination of NONHOlONOMIC AGENTS In 3-D}

Consider a group of $N$ agents in the three-dimensional space. Our goal in this section is to design a control law for each agent such that it guarantees they reach the consensus set.

Each agent is capable of communicating some information with its neighbors, defined by (1). The neighborhood set of agent $i, \mathcal{N}_{i}$, is a set of agents that can share their headings and attitudes (orientation) 


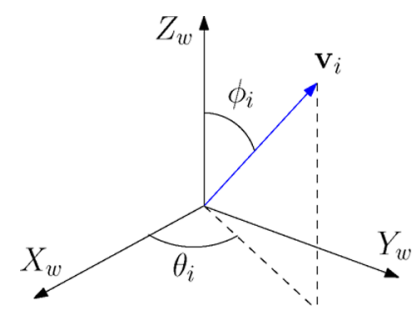

(a)

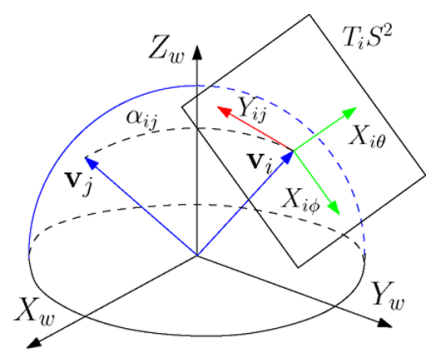

(b)
Fig. 2. (a) Heading of each agent is determined by two angles $\theta_{i}$ (heading) and $\phi_{i}$ (attitude) relative to the world frame. (b) Velocity vectors of agents in 3-D are projected onto a unit sphere. $X_{i \theta}$ and $X_{i \phi}$ form an orthonormal basis of the tangent plane. The geodesic versor $Y_{i j}$ points towards the geodesic direction from $\mathbf{v}_{i}$ to $\mathbf{v}_{j}$.

information with agent $i$. As before, it is assumed that there is a predetermined sphere with radius $R$ which determines the neighborhood relationship. The location of agent $i$ in the fixed world coordinates is given by $\left(x_{i}, y_{i}, z_{i}\right)$ and its velocity is $\mathbf{v}_{i}=\left(\dot{x}_{i}, \dot{y}_{i}, \dot{z}_{i}\right)^{T}$. The orientation of the velocity vector of agent $i$ can be characterized by specifying two angles $\theta_{i}$ (heading) and $\phi_{i}$ (attitude) relative to the world frame [see Fig. 2(a)], and they are defined as

$$
\begin{aligned}
& \theta_{i}=\operatorname{atan} 2\left(\dot{y}_{i}, \dot{x}_{i}\right), 0 \leq \theta_{i} \leq 2 \pi \\
& \phi_{i}=\operatorname{atan} 2\left(\sqrt{\dot{x}_{i}^{2}+\dot{y}_{i}^{2}}, \dot{z}_{i}\right), 0<\phi_{i}<\pi .
\end{aligned}
$$

Without loss of generality, it is assumed that all agents move with a constant unit speed. The velocity of agent $i$ in 3 dimensions is given by

$$
\mathbf{v}_{i}=\left(\begin{array}{c}
\dot{x}_{i} \\
\dot{y}_{i} \\
\dot{z}_{i}
\end{array}\right)=\left(\begin{array}{c}
\cos \theta_{i} \sin \phi_{i} \\
\sin \theta_{i} \sin \phi_{i} \\
\cos \phi_{i}
\end{array}\right)
$$

Hence, all velocity vectors are on a unit sphere $\mathbf{S}^{2} \doteq\{\mathbf{p}=(x, y, z) \in$ $\left.\mathbb{R}^{3}:\|\mathbf{p}\|=1\right\}$ [see Fig. 2(b)]. We represent each vector $\mathbf{v}_{i}$ as a point on this unit sphere. As the direction of the velocity vector of agent $i$ changes, the corresponding point $\mathbf{v}_{i}$ will move along a curve on the sphere. The tangent vector to this curve at $\mathbf{v}_{i} \in \mathbf{S}^{2}$ can be uniquely represented as a vector $\dot{\mathbf{v}}_{i} \in \mathbb{R}^{3}$ such that $\dot{\mathbf{v}}_{i} \perp \mathbf{v}_{i}$ and $\dot{\mathbf{v}}_{i} \in T_{i} \mathbf{S}^{2}$ where $T_{i} \mathbf{S}^{2}$ is the tangent plane at $\mathbf{v}_{i}$. A basis for the tangent space $T_{i} \mathbf{S}^{2}$ can be obtained by differentiating $\mathbf{v}_{i}$, and thus $\dot{\mathbf{v}}_{i}$ can be written as

$$
\dot{\mathbf{v}}_{i}=u_{i \theta} X_{i \theta}+u_{i \phi} X_{i \phi} \in T_{i} \mathbf{S}^{2}
$$

where $\mathbf{B}_{i}=\left\{X_{i \theta}, X_{i \phi}\right\}$ is an orthonormal basis for the tangent plane $T_{i} \mathbf{S}^{2}$, and

$$
X_{i \theta}=\left(\begin{array}{c}
-\sin \theta_{i} \\
\cos \theta_{i} \\
0
\end{array}\right), X_{i \phi}=\left(\begin{array}{c}
\cos \theta_{i} \cos \phi_{i} \\
\sin \theta_{i} \cos \phi_{i} \\
-\sin \phi_{i}
\end{array}\right) .
$$

The control inputs $u_{i \theta}$ and $u_{i \phi}$ are related to $\dot{\theta}_{i}$ and $\dot{\phi}_{i}$ :

$$
u_{i \theta}=\dot{\theta}_{i} \sin \phi_{i} \quad u_{i \phi}=\dot{\phi}_{i}
$$

When points $\mathbf{v}_{i}$ and $\mathbf{v}_{j}$ are neither equal nor opposite, a vector $Y_{i j} \in$ $T_{i} \mathbf{S}^{2}$ called the geodesic versor can be defined to show the geodesic direction from $\mathbf{v}_{i}$ to $\mathbf{v}_{j}$ [see Fig. 2(b)]. The unit length geodesic versor is defined by (4). The difference from the 2-dimensional case is that on the sphere the angle $\alpha_{i j}$ is the radian distance between points $\mathbf{v}_{i}$ and $\mathbf{v}_{j}$ over the great circle path.

Now, we can prove the following theorem for the geodesic control of the velocity vectors of a group of $N$ agents, which is a generalization of Theorem 2 in [2] to an arbitrary number of agents and connected topologies.

Theorem 5.1: Consider the system of $N$ equations $\dot{\mathbf{v}}_{i}=u_{i \theta} X_{i \theta}+$ $u_{i \phi} X_{i \phi}, i=1, \ldots, N$. If the proximity graph of the agents is fixed and connected, then by applying the control laws

$$
\begin{aligned}
& u_{i \theta}=\sum_{j \in \mathcal{N}_{i}} \sin \alpha_{i j}\left\langle Y_{i j}, X_{i \theta}\right\rangle=\sum_{j \in \mathcal{N}_{i}}\left\langle\mathbf{v}_{j}, X_{i \theta}\right\rangle \\
& u_{i \phi}=\sum_{j \in \mathcal{N}_{i}} \sin \alpha_{i j}\left\langle Y_{i j}, X_{i \phi}\right\rangle=\sum_{j \in \mathcal{N}_{i}}\left\langle\mathbf{v}_{j}, X_{i \phi}\right\rangle
\end{aligned}
$$

all trajectories converge to the equilibria given by $u_{i \theta}=0$ and $u_{i \phi}=$ 0 , for $i=1, \ldots, N$. Furthermore, the consensus set is locally attractive.

Proof: Convergence to equilibria can be established using the Lyapunov function

$$
V=\frac{1}{2} \sum_{j \sim i}\left\|\mathbf{v}_{i}-\mathbf{v}_{j}\right\|^{2}=\sum_{j \sim i} 1-\left\langle\mathbf{v}_{i}, \mathbf{v}_{j}\right\rangle
$$

which is a measure of discrepancy among the velocity vectors. The time derivative of $V$ becomes

$$
\dot{V}=-\sum_{i=1}^{N} \sum_{j \in \mathcal{N}_{i}}\left\langle\dot{\mathbf{v}}_{i}, \mathbf{v}_{j}\right\rangle=-\sum_{i=1}^{N}\left(u_{i \theta}^{2}+u_{i \phi}^{2}\right) \leq 0 .
$$

Similar to the 2-D case, the configuration space (which is now an N copies of a sphere) is compact and therefore LaSalle's invariance principle can be used to establish convergence of all trajectories to invariant sets, including the synchronized state where all $\theta_{i}$ 's are the same and all $\phi_{i}$ 's are the same.

To prove local attractivity of the consensus set for the system of $\mathrm{N}$ agents with the control laws given in Theorem (5.1), we need to write (15) and (16) in terms of the heading and attitude angles. Using (14) we obtain these expressions for $\dot{\theta}_{i}$ and $\dot{\phi}_{i}$

$$
\begin{aligned}
\dot{\theta}_{i} & =-\sum_{j \in \mathcal{N}_{i}} \frac{\sin \phi_{j}}{\sin \phi_{i}} \sin \left(\theta_{i}-\theta_{j}\right) \\
\dot{\phi}_{i} & =-\sum_{j \in \mathcal{N}_{i}} \sin \left(\phi_{i}-\phi_{j}\right)-\sin \phi_{j} \cos \phi_{i}\left(1-\cos \left(\theta_{i}-\theta_{j}\right)\right)
\end{aligned}
$$

We now linearize (18) and (19) around the synchronized state $\phi_{i}=$ $\phi_{j}, \theta_{i}=\theta_{j}$. Let $\tilde{\theta}_{i}$ and $\tilde{\phi}_{i}$ be the deviations of $\theta_{i}$ and $\phi_{i}$ from the synchronized state. The linearized dynamics can be written as

$$
\dot{\tilde{\theta}}=-\sum_{j \in \mathcal{N}_{i}}\left(\tilde{\theta}_{i}-\tilde{\theta}_{j}\right) \quad \dot{\tilde{\phi}}_{i}=-\sum_{j \in \mathcal{N}_{i}}\left(\tilde{\phi}_{i}-\tilde{\phi}_{j}\right) .
$$

Now, consider the quadratic Lyapunov function

$$
V \doteq \frac{1}{2} \tilde{\theta}^{T} \tilde{\theta}+\frac{1}{2} \tilde{\phi}^{T} \tilde{\phi}
$$

Then, by using (20) we can show that $\dot{V}$ is nonpositive

$$
\dot{V}=\tilde{\theta}^{T} \dot{\tilde{\theta}}+\tilde{\phi}^{T} \dot{\tilde{\phi}}=-\tilde{\theta}^{T} L \tilde{\theta}-\tilde{\phi}^{T} L \tilde{\phi} \leq 0
$$




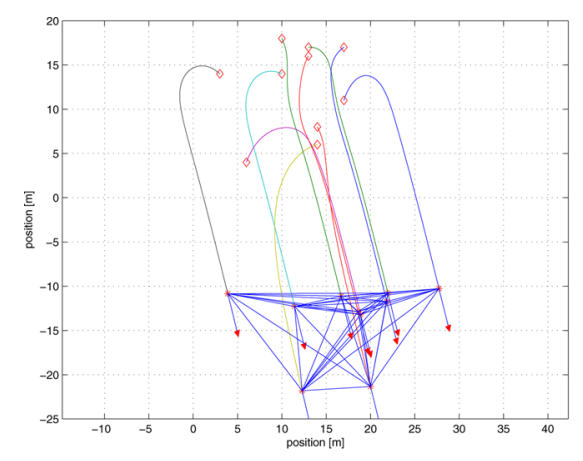

(a)

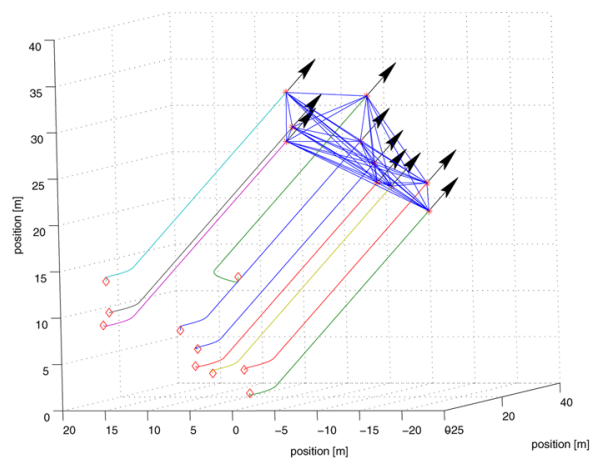

(b)

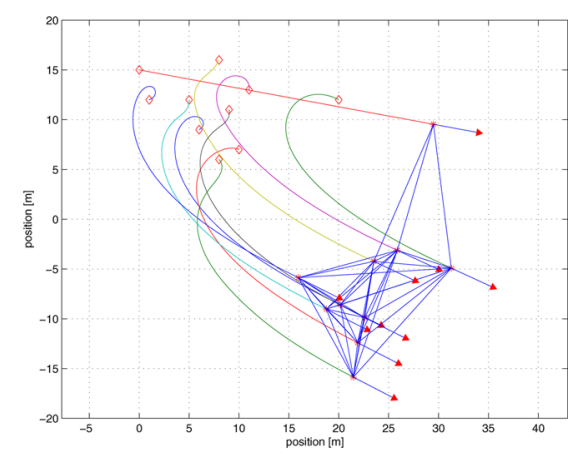

(c)

Fig. 3. Geodesic control laws result in (a) flocking in 2-D, (b) flocking in 3-D, and (c) following the leader in a planar motion.

where $L=B B^{T}$ is the graph Laplacian. Consider the compact set $\Omega_{c}=\{(\tilde{\theta}, \tilde{\phi}) \mid V \leq c\}$. By LaSalle's invariance principle any trajectory starting in $\Omega_{c}$ converges to the largest invariant set, $S$, contained in $E=\{(\tilde{\theta}, \tilde{\phi}) \mid \dot{V}=0\}$. The invariant set of this system is when $L \tilde{\theta}=0$ and $L \tilde{\phi}=0$, or when $\tilde{\theta}, \tilde{\phi} \in \operatorname{Null}\{L\}$. Since the graph is assumed to be connected, $\tilde{\phi}, \tilde{\theta} \in \operatorname{span}\{\mathbf{1}\}$ is the only invariant set. As a result, the synchronized state is locally asymptotically stable.

This analysis shows that with applying geodesic controllers (15) and (16) the consensus set is locally attractive.

\section{StABILITY ANALYSIS FOR SWITCHING GRAPHS IN 2 DimENSIONS}

So far, the underlying assumption has been that the graph $\mathbb{G}$, representing the neighborhood relationship, is fixed and connected. In practice, the motion of individual agents will result in change in topology. To avoid complications that occur because of discontinuous change in the set of nearest neighbors, we will assume that there is always a minimum time, called a dwell time, $\mu_{D}$, over which the graph does not change. This simplifying assumption will avoid infinite switches over a finite period of time.

Secondly, we assume that the switching is such that some weak notion of connectivity "persists" over finite time intervals. Each agent $i$ would use control laws similar to (5) (which is now hybrid, since the set of neighbors $\mathcal{N}_{i}$ changes discontinuously). With the dwell time assumption, the control inputs would be of the following form:

$$
\omega_{i}(t)=\sum_{j \in \mathcal{N}_{i}\left(t_{k}\right)}\left\langle\mathbf{v}_{j}(t), X_{i \theta}(t)\right\rangle, t \in\left[t_{k}, t_{k}+\mu_{D}\right) .
$$

The hybrid controller (22) may result in change of the proximity graph as the switching occurs. Let $\mathcal{P}$ denote a set that indexes the class of all simple graphs defined on $N$ vertices; so if $p \in \mathcal{P}$ then $\mathbb{G}_{p}$ is the corresponding proximity graph on $N$ vertices. Let $\sigma(t)$ be a piecewise constant switching signal whose value at time $t$ is the index of the graph representing the proximity graph of agent $i$. As mentioned earlier, following [5], we need to define a weaker notion of connectivity for a collection of graphs with a switching signal $\sigma$ with $\sigma\left(t_{k}\right)=p_{k}$.

Definition 6.1: A collection of graphs is called jointly connected, if the union of its members is a connected graph.

It is natural to say that the $N$ agents under consideration are linked together across a time interval $[t, \tau]$ if the collection of graphs $\left\{\mathbb{G}_{\sigma\left(t_{k}\right)}, \mathbb{G}_{\sigma\left(t_{k+1}\right)}, \ldots, \mathbb{G}_{\sigma(\tau)}\right\}$ encountered along the interval, is jointly connected.

In trying to extend Theorem 3.3 to graphs with the aforementioned switching regime, we need the following lemma, which was proved in [5].
Lemma 6.2: If $\left\{\mathbb{G}_{p_{1}}, \mathbb{G}_{p_{2}}, \ldots, \mathbb{G}_{p_{m}}\right\}$ is a jointly connected collection of graphs with Laplacians $L_{p_{1}}, L_{p_{2}}, \ldots, L_{p_{m}}$, then

$$
\bigcap_{i=1}^{m} \operatorname{kernel} L_{p_{i}}=\operatorname{span}\{\mathbf{1}\} .
$$

The previous lemma states that the intersection of the null space of the Laplacians of a set of jointly connected graphs is only the vector of ones. We can now state the following theorem.

Theorem 6.3: Let $\mu_{D}>0$ and the initial heading vector $\theta_{0}$ be fixed. Let $\sigma:[0, \infty) \rightarrow \mathcal{P}$ be a piecewise constant persistent switching signal corresponding to all graphs over $N$ vertices whose switching times $t_{1}, t_{2}, \ldots$ satisfy $\left(t_{k+1}-t_{k}\right) \geq \mu_{D}, k \geq 1$. If there exists an infinite sequence of non-empty, bounded, time-intervals with the property that across each such interval the $N$-agent group is linked together, then by applying geodesic control law (22) all trajectories converge to the equilibria of $\omega_{i}(t)=0$. Furthermore, the consensus set is locally attractive.

The proof of convergence to the consensus set is omitted due to space limitations, but the idea here is to use Lemma 6.2 in the context of a LaSalle-like invariance principle for switched systems with dwell time constraint on switching. With the assumption that there exists an infinite sequence of nonempty, bounded, time-intervals with the property that across each such interval the graphs are jointly connected, we show that the intersection of the null spaces of the corresponding Laplacians is only the vector of ones. In other words, even though the graphs might be disconnected, and as a result their Laplacians have a larger kernel, the intersection is span $\{\mathbf{1}\}$. By showing that the heading angles follow the persistent direction in the intersection of the Laplacians, we conclude that the consensus set is locally attractive. (cf. [11] for the detailed description of the proof).

\section{Simulations}

In this section, we numerically show that the distributed control law (5), for the planar case, and the geodesic control laws (15) and (16), for the three-dimensional case, can force a group of agents to reach the consensus state. Fig. 3(a) and (b) show the leaderless flocking of ten agents in two and three dimensions, respectively. The initial position and heading of all agents are generated randomly within a prespecified area. The neighboring radius is chosen large enough so that agents form a connected graph at time $t=0$. The arrows on each agent show the directions of the velocity vectors. Simulations show that agents smoothly adjust their headings and after a reasonable amount of time they converge to a formation, and their relative distances stabilizes. 
Fig. 3(c) shows the effect of the presence of a leader in the group. In the simulations, one of the agents is randomly chosen to be the leader of the group, and its heading is constant. Without knowing which one of them is the leader, all other agents adjust their headings to follow him so that the formation remains stable. Even if the leader's motion has dynamics, as long as the group remains connected, all agents follow the leader.

\section{CONCLUSION AND FUTURE WORK}

We provided a coordination scheme which resulted in flocking of a collection of kinematic agents. The control law was based on nearest neighbor sensing. It was shown that reaching consensus is possible despite possible changes in the topology of the proximity graph representing the neighborhood relationship. A generalization of the current analysis would be to develop results similar to [15], [16] for dynamic models, by using artificial potential functions similar to [13]. An important question that we need to answer is how to enforce the connectivity condition of the proximity graph. A potential starting point would be to use results of [20] in topology control.

\section{REFERENCES}

[1] R. W. Beard and V. Stepanyan, "Synchronization of information in distributed multiple vehicle coordinated control," in Proc. IEEE Conf. Decision Control, Dec. 2003, pp. 2029-2034.

[2] F. Bullo, R. M. Murray, and A. Sarti, Control on the sphere and reduced attitude stabilization Calif. Inst. Technol., Pasadena, CA, 1995, Tech. Rep. CaltechCDSTR:1995.CIT-CDS-95-005.

[3] J. P. Desai, J. P. Ostrowski, and V. Kumar, "Modeling and control of formations of nonholonomic mobile robots," IEEE Trans. Robot. Automat., vol. 17, no. 6, pp. 905-908, Dec. 2001.

[4] R. Horn and C. Johnson, Matrix Analysis. Cambridge, U.K.: Cambridge Univ. Press, 1999.

[5] A. Jadbabaie, J. Lin, and A. S. Morse, "Coordination of groups of mobile autonomous agents using nearest neighbor rules," IEEE Trans. Autom. Control, vol. 48, no. 6, pp. 988-1001, Jun. 2003.

[6] A. Jadbabaie, N. Motee, and M. Barahona, "On the stability of $\mathrm{Ku}-$ ramoto model of coupled nonlinear oscillators," in Proc. Amer. Control Conf., Jun. 2004, pp. 4296-4301.

[7] J. Jeane, N. E. Leonard, and D. Paley, "Collective motion of ringcoupled planar particles," in Proc. 44th IEEE Conf. Decision Control, Seville, Spain, Dec. 2005, pp. 3929-3934.

[8] E. W. Justh and P. S. Krishnaprasad, "Equilibria and steering laws for planar formations," Syst. Control Lett., vol. 52, no. 1, pp. 25-38, May 2004.

[9] Z. Lin, M. Brouke, and B. Francis, "Local control strategies for groups of mobile autonomous agents," IEEE Trans. Autom. Control, vol. 49, no. 4, pp. 622-629, Apr. 2004.

[10] L. Moreau, "Stability of multiagent systems with time-dependent communication links," IEEE Trans. Autom. Control, vol. 50, no. 2, pp. 169-182, Feb. 2005.

[11] N. Moshtagh and A. Jadbabaie, Distributed geodesic control laws for flocking of nonholonomic agents [Online]. Available: http://www.seas. upenn.edu/ jadbabai/papers/tech-note-geodesic-2col-final.pdf

[12] R. Olfati-Saber and R. M. Murray, "Consensus problems in networks of agents with switching topology and time-delays," IEEE Trans. Autom. Control, vol. 49, no. 9, pp. 1520-1533, Sep. 2004.

[13] N. E. Leonard, P. Ogren, and E. Fiorelli, "Cooperative control of mobile sensing networks: Adaptive gradient climbing in a distributed environment," IEEE Trans. Autom. Control, vol. 49, no. 8, pp. 1292-1302, Aug. 2004.

[14] S. H. Strogatz, "From Kuramoto to Crawford: Exploring the onset of synchronization in population sof coupled nonlinear oscillators," Physica D, vol. 143, pp. 1-20, 2000.

[15] H. G. Tanner, A. Jadbabaie, and G. J. Pappas, "Flocking in teams of nonholonomic agents," in Cooperative Control, ser. Springer Series in Control and Informations Science, S. Morse, N. Leonard, and V. Kumar, Eds. New York: Springer-Verlag, 2004, vol. 309, pp. 229-239, accepted for publication.

[16] _ _ "Flocking in fixed and switching newtorks," IEEE Trans. Autom. Control, 2007, to be published.
[17] J. N. Tsitsiklis, "Problems in decentralized decision making and computation," Ph.D. dissertation, Dept. EECS, MIT, Cambridge, MA, 1984.

[18] T. Vicsek, A. Czirok, E. B. Jacob, I. Cohen, and O. Schochet, "Novel type of phase transitions in a system of self-driven particles," Phys. Rev. Lett., vol. 75, pp. 1226-1229, 1995.

[19] W. Wang and J. J. E. Slotine, On partial contraction analysis for couplled nonlinear oscillators. Tech. Rep. MIT, Nonlinear Syst. Lab., Cambridge, MA, 2003.

[20] R. Wattenhofer, L. Li, P. Bahl, and Y. Wang, "Distributed topology control for wireless multihop ad-hoc networks," in Proc. INFOCOM, 2001, pp. 1388-1397.

\section{Fundamental Constraints on Uncertainty Evolution in Hamiltonian Systems}

Fu-Yuen Hsiao and Daniel J. Scheeres

Abstract-A realization of Gromov's nonsqueezing theorem and its applications to uncertainty analysis in Hamiltonian systems are studied in this note. Gromov's nonsqueezing theorem describes a fundamental property of symplectic manifolds, however, this theorem is usually started in terms of topology and its physical meaning is vague. In this note we introduce a physical interpretation of the linear symplectic width, which is the lower bound in the nonsqueezing theorem, in terms of the eigenstructure of a positive-definite, symmetric matrix. Since uncertainty is often represented in terms of a positive definite, symmetric matrix in control theory, our study can be applied to uncertainty analysis by applying the nonsqueezing theorem to the uncertainty ellipsoid. We find a fundamental inequality for the evolving uncertainty in a linear dynamical system and provide some numerical examples.

Index Terms-Hamiltonian system, linear symplectic width, nonsqueezing theorem, symplectic manifold, uncertainty analysis.

\section{INTRODUCTION}

In this note, we study the realization of Gromov's nonsqueezing theorem [3] in Hamiltonian systems and apply our results to uncertainty analysis. An example from orbit determination of spacecraft is given as a potential application. The nonsqueezing theorem was first proved by Gromov using $J$-holomorphic curves [3]. Other mathematicians have given proofs using different approaches [4], [8]. The theorem was then extended to arbitrary symplectic manifolds by Lalonde and McDuff [5]. Although this theorem has been proven rigorously in a topological sense, its application to practical issues is still vague.

A well-known result from Hamiltonian dynamical systems theory is Liouville's Theorem [1], which says that the "volume" of a phase flow in a nondissipative system is conserved. This result also applies to un-

Manuscript received June 2, 2005; revised May 23, 2006 and November 21, 2006. Recommended by Associate Editor J. Berg. This work was supported in part by the national Science Council of Taiwan under Project NSC-96-2623-7032-001-D.

F.-Y. Hsiao is with the Department of Aerospace Engineering, Tamkang University, Tamsui 251, Taiwan, R.O.C. (e-mail: fyhsiao@gmail.edu).

D. J. Scheeres is with the Department of Aerospace Engineering, The University of Michigan, Ann Arbor, MI 48109-2140 USA (e-mail: scheeres@umich. edu).

Digital Object Identifier 10.1109/TAC.2007.894531 